\title{
Electron-antineutrino disappearance seen by Daya Bay reactor neutrino experiment
}

\author{
Ruiguang Wang, on behalf of the Daya Bay Collaboration
}

Institute of High Energy Physics, Beijing, 100049, China

email: wangrg@mail.ihep.ac.cn.

\begin{abstract}
The Daya Bay Reactor Neutrino Experiment has measured a non-zero value for the neutrino mixing angle $\theta_{13}$ with a significance of 7.7 standard deviations. Antineutrinos from six $2.9 \mathrm{GW}_{t h}$ reactors were detected in six antineutrino detectors deployed in two near and one far underground experimental halls. With a 116.8 kton-GW Gh $_{t h}$ day live-time exposure in 139 days, $28,909(205,308)$ electron-antineutrino candidates were detected at the far hall (near hall). The ratio of the observed to expected number of antineutrinos at the far hall is $\mathrm{R}=0.944 \pm 0.007$ \pm 0.003 (syst). A rate-only analysis finds $\sin ^{2} 2 \theta_{13}=0.089 \pm 0.010$ (stat) \pm 0.005 (syst) in a three-neutrino framework.
\end{abstract}

Keywords. Neutrino oscillation, reactor neutrino, mixing angle $\theta_{13}$, Daya Bay

\section{Introduction}

It is well known that the flavor of neutrinos oscillate as they propagate. Neutrino oscillations can be described by the three mixing angles $\left(\theta_{12}, \theta_{23}, \theta_{13}\right)$ and a phase of the PMNS matrix, and two mass-squared differences $\left(\Delta m_{32}\right.$ and $\left.\Delta m_{21}\right)$. Of these mixing angles, $\theta_{13}$ is the least known. Some experiments, such as CHOOZ, T2K, MINOS and Double CHOOZ, have indicated that $\theta_{13}$ could be nonzero. In March 2012, the Daya Bay collaboration first announced the discovery of a non-zero $\theta_{13}$ with more than $5 \sigma$ (An et al. 2012a). Several months later this value was improved to $7.7 \sigma$ (An et al. 2013).

In reactor based experiments, the precise $\theta_{13}$ can be determined via the survival probability of the electron-antineutrino $\bar{\nu}_{e}$ at short distances from the reactors,

$$
P_{\text {sur }} \approx 1-\sin ^{2} 2 \theta_{13} \sin ^{2}\left(1.267 \Delta m_{31}^{2} L / E\right),
$$

where $\Delta m_{31}^{2}=\Delta m_{32}^{2} \pm \Delta m_{21}^{2}, E$ is the $\bar{\nu}_{e}$ energy in $\mathrm{MeV}$ and $L$ is the distance in meters between the $\bar{\nu}_{e}$ source and the detector (baseline). The near-far arrangement of Daya Bay identical antineutrino detectors (ADs), as illustrated in Figure 1, allows for a relative measurement by comparing the observed $\bar{\nu}_{e}$ rates at various baselines with minimal uncertainties.

\section{The experiment}

The Daya Bay experiment sets up at the Daya Bay nuclear power complex in Shenzhen, south China. Six identical reactor cores spaced in pairs provide a total $17.4 \mathrm{GW}_{t h}$ thermal power. Three underground experimental halls (EHs) are connected with about $3,000 \mathrm{~m}$ horizontal tunnels. EHs' overburdens are 250, 265 and 860 (m.w.e). The baselines from six ADs to six cores are precisely measured by GPS. The baseline uncertainties have no effect on the oscillation results.

As shown in figure 2, each $\mathrm{AD}$ has three coaxial cylindrical vessels, $3 \mathrm{~m}$ inner (IAV) and $4 \mathrm{~m}$ outer (OAV) acrylic vessels and $5 \mathrm{~m}$ stainless steel vessel (SSV), filled with $20 \mathrm{t}$ 


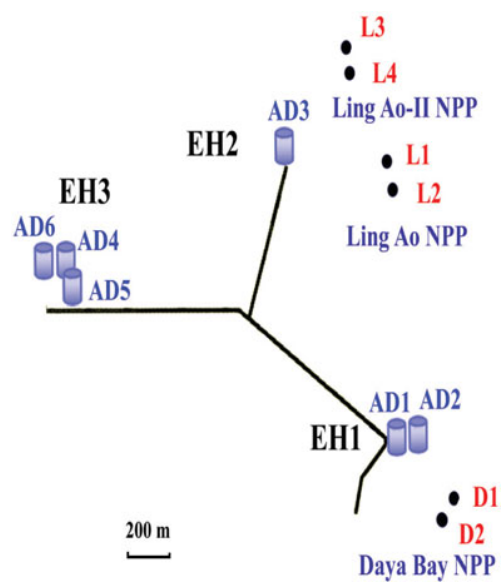

Figure 1. Layout of the Daya Bay experiment. The dots represent reactors, labeled as D1, D2, L1, L2, L3 and L4. Six ADs, AD1-AD6, are installed in three EHs.

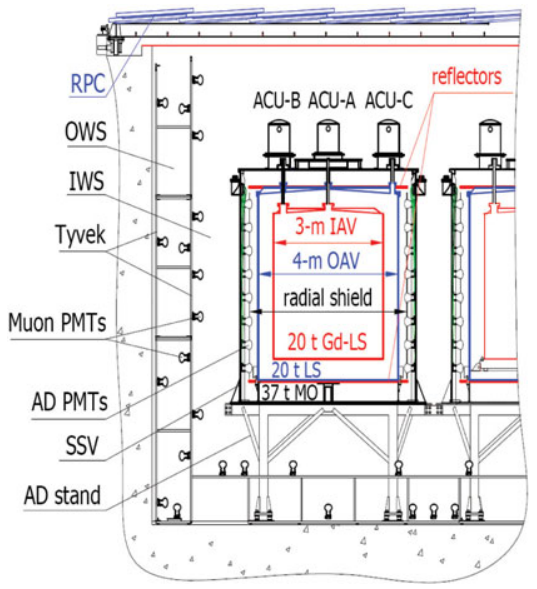

Figure 2. The Daya Bay detectors, consisting of ADs (IAV, OAV,SSV), calibration units (ACU-A, ACU-B, and ACU-C), muon system (RPC, OWS, IWS) and PMTs.

of $0.1 \%$ by weight gadolinium-doped liquid scintillator (Gd-LS), $21 \mathrm{t}$ of un-doped liquid scintillator (LS) and $37 \mathrm{t}$ of mineral oil (MO), respectively. Three automated calibration units (ACU-A, ACU-B, and ACU-C) are mounted at the top of each SSV. Each ACU is equipped with a LED, a ${ }^{68} \mathrm{Ge}$ source, and a combined source of ${ }^{241} \mathrm{Am}-{ }^{13} \mathrm{C}$ and ${ }^{60} \mathrm{Co}$. The muon detection system consists of a resistive plate chamber (RPC) tracker and a high-purity active inner (IWS) and outer (OWS) water shields. There are 1928 -inch Hamamatsu PMTs installed in each AD and 288 (384) PMTs installed in each near (far) hall water pool. A detailed description of the Daya Bay experiment can be found in (An et al. 2012b).

\section{Data analysis and results}

This analysis is based on data from December 24, 2011 to May 11, 2012. The $\bar{\nu}_{e} \mathrm{~s}$ are detected via the inverse $\beta$-decay (IBD) reaction, $\bar{\nu}_{e}+p \rightarrow e^{+}+n$, in Gd-LS. The coincidence of the prompt scintillation from the $e^{+}$and the delayed neutron capture on Gd provides a distinctive $\bar{\nu}_{e}$ signature. The IBD selection includes: 1) prompt energy $0.7 \sim 12 \mathrm{MeV}$ and delayed energy $6 \sim 12 \mathrm{MeV}, 2)$ prompt-delayed signals time duration $<200 \mu$ s and 3) no triggers $200 \mu$ s before prompt or after delayed event. The contribution of IBD-like events is estimated less than $0.3 \%$. The detector efficiencies as well as correlated and uncorrelated systematic uncertainties are also estimated as $78.8 \%, 1.9 \%$ and $0.2 \%$, respectively.

The $\bar{\nu}_{e}$ rate in the far hall was predicted with a weighted combination of the two near hall measurements assuming no oscillation. The deficit observed at the far hall is $R=0.944 \pm 0.007$ (stat) \pm 0.003 (syst). The value of $\sin ^{2} 2 \theta_{13}$ was determined with a $\chi^{2}$ constructed with pull terms accounting for the correlation of the systematic errors, which is $\sin ^{2} 2 \theta_{13}=0.089 \pm 0.010$ (stat.) \pm 0.005 (syst.) with a $\chi^{2} / \mathrm{NDF}$ of $3.4 / 4$. All best estimates of pull parameters are within its one standard deviation based on the corresponding systematic uncertainties. The no-oscillation hypothesis is excluded at 7.7 standard deviations (in figure 3 ). The observed $\bar{\nu}_{e}$ spectrum in the far hall was compared to a prediction based on the near hall measurements is shown in Figure 4. The distortion 


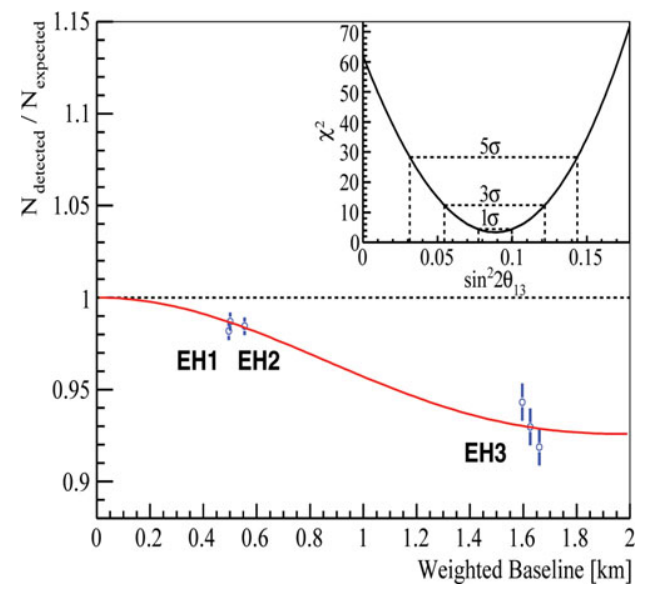

Figure 3. Ratio of measured versus expected signal in each detector, assuming no oscillation. The error bar is the uncorrelated uncertainty of each $\mathrm{AD}$, including statistical, detector-related, and background-related uncertainties. The expected signal was corrected with the best-fit normalization parameter. Reactor and survey data were used to compute the flux-weighted average baselines. The oscillation survival probability at the best-fit value is given by the smooth curve. The $\chi^{2}$ versus $\sin ^{2} 2 \theta_{13}$ is shown in the inset.

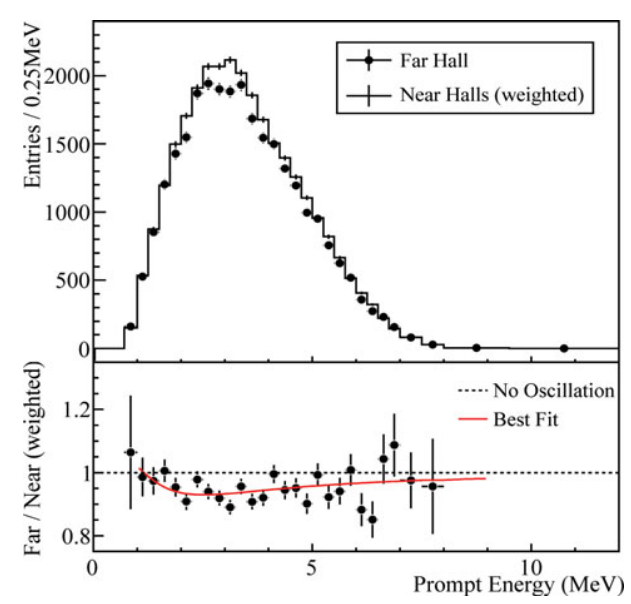

Figure 4. Top: Measured prompt energy spectrum of the far hall (sum of three ADs) compared with the no-oscillation prediction from the measurements of the two near halls. Bottom: The ratio of measured and predicted no-oscillation spectra. The red curve is the best-fit solution with $\sin ^{2} 2 \theta_{13}=0.089$ obtained from the rate-only analysis. The dashed line is the no-oscillation prediction.

of the spectra is consistent with the expected one calculated with the best-fit $\theta_{13}$ obtained from the rate-only analysis, providing further evidence of neutrino oscillation.

Up to now Daya Bay experiment has obtained the most precise value of $\sin ^{2} 2 \theta_{13}$ in the world. After installation of final two ADs in the summer of 2012, running for several years with eight ADs will yield even better results.

\section{References}

An, F. P. et al. (Daya Bay Collaboration) 2012a, Phys. Rev. Lett., 108, 171803

An, F. P. et al. (Daya Bay Collaboration) 2012b, Nucl. Instr. and Meth., A685, 78

An, F. P. et al. (Daya Bay Collaboration) 2013, Chinese Phys. C, 3, 7, 011001 